\title{
Simplified back-propagation for fibre nonlinearity compensation employing multi-band OFDM signals
}

\author{
L. M. Pessoa - H. M. Salgado - I. \\ Darwazeh
}

Received: date / Accepted: date

\begin{abstract}
In this paper we investigate the efficacy of applying the coupled field back-propagation algorithm as a post-compensation method for nonlinearity mitigation in a coherently detected fibre optic long-haul system using multi-band orthogonal frequency division multiplexed signals. Specifically, we analyze the impact of varying the band-spacing and the number of bands. We find that its efficacy is higher for largely spaced bands and a small number of bands. Additionally, we propose a method to include the Four Wave Mixing compensating term within the coupled field method to provide simplified means of multichannel compensation. We conclude that this method is more efficient in improving the performance especially for small band spacings. The coupled field method proves to be an interesting choice for the implementation of receiver-based real-time digital signal processing.
\end{abstract}

Keywords Multi-band OFDM · Back-propagation · Four wave mixing

\section{Introduction}

The interplay between fibre chromatic dispersion, intra-channel nonlinearities and amplifier noise is determinant in limiting the maximum capacity of a fibre-optic long haul transmission. However, when considering the transition from single channel to multichannel (Wavelength Division Multiplexed - WDM) systems, attention must be paid also to nonlinear inter-channel effects, where the nonlinearity is no longer stemming only from Self Phase Modulation (SPM) but also from Cross Phase Modulation (XPM) and Four Wave Mixing (FWM) effects. Traditionally,

L. M. Pessoa · H. M. Salgado

INESC TEC (formerly INESC Porto) and Faculty of Engineering, University of Porto, 4200-465 - Porto, Portugal

E-mail: luis.m.pessoa@inescporto.pt, hsalgado@inescporto.pt

I. Darwazeh

Department of Electronic and Electrical Engineering, University College London

Torrington Place - London WC1E 7JE, U.K.

E-mail: i.darwazeh@ee.ucl.ac.uk 
the optical communications community has solved the problem of inter-channel nonlinearities resorting to dispersion management and mid-span phase conjugation techniques, as shown in Chowdhury and Essiambre (2004). More recently, increasing attention has been put into electronic impairment compensation techniques, either using pre or post compensation, as discussed in Lowery (2007); Roberts et al. (2006), essentially due to the progress in coherent optical detection, analogto-digital - ADC (for post-compensation) and digital-to-analog - DAC (for precompensation) devices, associated with digital signal processing techniques, which are the building blocks for impairment compensation in the electronic domain. In this context, the back-propagation (BP) algorithm, proposed independently by Li et al. (2008) and Ip and Kahn (2008), has been gaining increasing interest. BP consists in passing the received signal (in the case of post-compensation) or the transmitted signal (in the case of pre-compensation), through a virtual fibre with opposite signs of dispersion and nonlinearity, yielding an estimate of the originally transmitted signal. In the absence of noise and limited computation power and provided the characteristics of the transmission channel are known, this technique can recover exactly the signal that was transmitted, as shown by Ip and Kahn (2008). In fact, only approximate solutions can be attained due to practical noise and computational complexity limits.

The present paper concentrates on post compensation techniques assuming coherent detection, focusing on multichannel back-propagation using a single polarization transmission. Furthermore, we focus on point-to-point links, since the dynamic nature of signal routing in mesh networks would not be compatible with techniques exploring the inter-channel interaction. Therefore, although not feasible in such mesh network scenarios, the presented multi-channel compensation techniques (and possible improvements) are likely to become widely used in future point-to-point long haul fiber-optic deployments, due to their reduced implementation complexity. The topic of multichannel back-propagation will be addressed considering orthogonal frequency division multiplexed (OFDM) signals having multiple bands. The computational complexity and performance of two different BP implementations will be compared, namely the total field (TF) approach, introduced by Ip and Kahn (2009); Li et al. (2008), and the coupled field (CF) approach, which consists of a simplification introduced by Mateo et al. (2008). While in the former, the full electric field of the multi-band signal is fed to the BP algorithm, in the latter, each band is back-propagated separately while including the contributions from other bands. We should also observe that while polarization multiplexing is being considered in both current and future system deployments, the current work focus only on single polarization systems for the sake of simplicity. However, we have determined through computer simulation that the discussed algorithms and techniques are also suitable for polarization multiplexed transmission. Such extended analysis can be performed by using the Manakov equation described in Agrawal (2001), which takes into account the interactions between the orthogonal polarizations. Furthermore, it should be noted that the Manakov equation assumes that the relative orientations of polarizations among the several channels remains unchanged during propagation, which can only be assumed if the bandwidth of the total field in propagation is narrow enough to allow the polarization mode dispersion to be negligible.

Although previous works have generically compared the CF and TF approaches, such as Mateo and Li (2009); Mateo et al. (2008), the efficacy of applying these 
simplified techniques to multi-band OFDM signals remains unknown. Furthermore, in order to fully understand the circumstances under which the efficacy of this simplification techniques might be beneficial, we also investigate their performance for variable band spacing and number of bands, which further justifies the significance of the present research. Considering the transmission of multi-band OFDM signals, we propose for the first time a method to include the FWM compensating term within the coupled field method to provide simplified means of multichannel compensation. This method is suitable for a small number of OFDM bands, being able to provide an improvement in the performance while maintaining its computational complexity. The remainder of this paper is organized as follows: section 2 describes the simulation environment and introduces the $\mathrm{CF}$ and TF back-propagation algorithms. In section 3, through the analysis of simulation results, the computational complexity of both $\mathrm{CF}$ and $\mathrm{TF}$ approaches is compared for both variable band-spacing and number of bands. Furthermore, in section 4, the coupled field algorithm will be extended to include the effect of Four Wave Mixing. Conclusions are given in section 5.

\section{Specification of simulation environment}

\subsection{General Specification}

The analysis presented in this paper considers a transmission setup composed by a multi-band OFDM optical transmitter (considering an ideal electro-optic converter), with a launched power of $+4 \mathrm{dBm}$, in order to operate in a nonlinear regime. This is followed by an optical fibre channel composed by 25 spans of $80 \mathrm{Km}$ SMF each, having a dispersion $D$ of $17 \mathrm{ps} /(\mathrm{nm}-\mathrm{km})$, a nonlinear parameter $\gamma$ of $0.0013 \mathrm{~m}^{-1} \mathrm{~W}^{-1}$, an attenuation $\alpha$ of $0.2 \mathrm{~dB} / \mathrm{km}$ and an amplifier with a noise figure of $5 \mathrm{~dB}$. At the receiver, we consider an ideal coherent optical receiver, followed by an antialiasing filter before the sampling process, which occurs at an oversampling rate of 3 samples per symbol. The antialiasing filter is set to a lowpass 5 th order Butterworth with a bandwidth of $40 \%$ of the oversampling rate.

\subsection{Multi-band OFDM}

One of the main challenges associated with the investigation of electronic compensation of optical system distortions has been the fact that the bandwidth requirements of state of the art research systems is always much higher than the what the best capabilities of ADC/DAC can meet. Shieh et al. (2008) has demonstrated that this electronics bottleneck can be overcome by using the concept of orthogonal band multiplexing to divide the entire OFDM spectrum into multiple orthogonal bands (orthogonal-band-multiplexed OFDM - OBM-OFDM). These orthogonal bands can coexist with a small or even zero guard band between them, since the orthogonality condition allows for band de/multiplexing without interband interference. In the OBM-OFDM approach, the entire OFDM spectrum is divided into $N$ OFDM bands, each having a subcarrier spacing $f_{d}$. Therefore, in order to achieve orthogonality between bands, it is enough to use a guard band that is an integer multiple of the subcarrier spacing $f_{d}$. In this way, each OFDM 
band is an orthogonal extension of another band. In the receiver, the laser should be tuned to the center of each band, and then the signal is filtered by an anti-alias filter having a bandwidth slightly larger than the band itself.

The OBM-OFDM modulation scheme adopted in this work was implemented in Matlab, having a total number of 128 subcarriers, QPSK encoding, a guardinterval of $1 / 8$ of the observation period, and the middle 88 subcarriers filled with information. Each OFDM band carries a 5 GSymbols/s stream of useful data. The total number of symbols considered in the simulation was set to $2^{14}$. The anti-alias filter used accommodates a bandwidth equivalent to the frequency spectrum occupied by 138 subcarriers, which gives the equivalent to 10 subcarriers of tolerance, since each band has 128 subcarriers.

\subsection{Back-propagation}

The propagation of a pulse $E(z, t)$ through a single mode optical fibre, for single polarization transmission, is governed by the nonlinear Schrödinger equation (NLSE) as shown in Agrawal (2001). The NLSE can be written in the following simplified manner:

$$
\frac{\partial E}{\partial z}=(\hat{D}+\hat{N}) E
$$

where $\hat{D}$ is a differential operator accounting for dispersion and attenuation, and $\hat{N}=j \gamma|E|^{2}$ is the nonlinear operator governing nonlinear effects in the propagating signal, with $\gamma$ representing the nonlinearity parameter of the optical fibre. The NLSE is typically solved numerically by the split-step Fourier (SSF) method introduced by Hasegawa and Tappert (1973). The numerical solution given by the SSF method involves dividing the optical fibre into small segments of length $h$, wherein the effects of dispersion and nonlinearity are considered independently, which is an approximation. Back-propagation is also implemented through the SSF method, while using inverse signs for the $\hat{D}$ and $\hat{N}$ operators. In this work we consider backpropagation using the asymmetric approximation for the SSF method, discussed in Hayee (2008); Ip and Kahn (2008), which consists in dividing the propagation from $z$ to $z+h$ into two steps, one for nonlinearity and another for dispersion. However, forward propagation simulations are performed using the more precise symmetric approximation proposed by Agrawal (2001). This is achieved using a small adaptive step size $h$ in order to minimize the error, by satisfying the step size requirements indicated by Hayee (2008).

\subsubsection{Total field Back-propagation}

When considering a generic WDM multichannel signal, its bandwidth is generally much larger than the electrical bandwidth of a single photo-receiver. Additionally, not only the photodiode is a restriction factor but also the analogue to digital converter, whose sample rate is not enough to accommodate the whole bandwidth of a WDM signal. Therefore a structure composed by a bank of local oscillators tuned to the center frequencies of each of the channels of the WDM signal, is required in order to translate each of those channels to baseband. Then, the full electric field of the WDM signal $(E)$ might be reconstructed through a coherent 
sum of each baseband signal $\left(\hat{E}_{m}\right)$ such that $E=\sum_{m=1}^{N} \hat{E}_{m} \exp (j m \Delta \omega t)$, where $N$ represents the number of channels and $\Delta \omega$ represents the angular frequency of channel separation. The reconstructed optical field can be used as an input to the back-propagation algorithm in order to compensate for the transmission impairments, as shown in Fig. 1(a), which has been named the total field algorithm, as described in Ip and Kahn (2009); Li et al. (2008). Since the full electric field is taken into account, it compensates for all SPM, XPM and FWM effects.

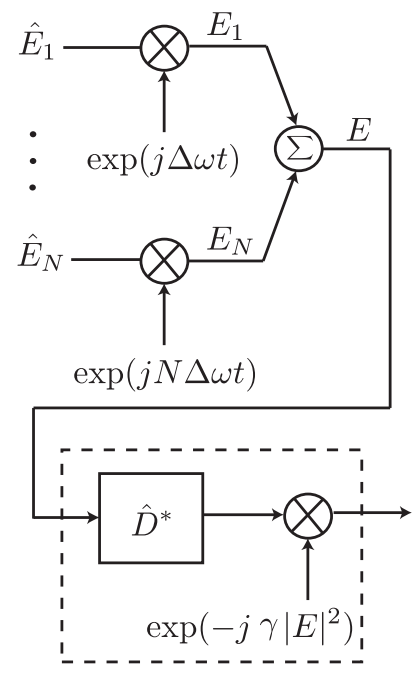

(a) Total Field BP

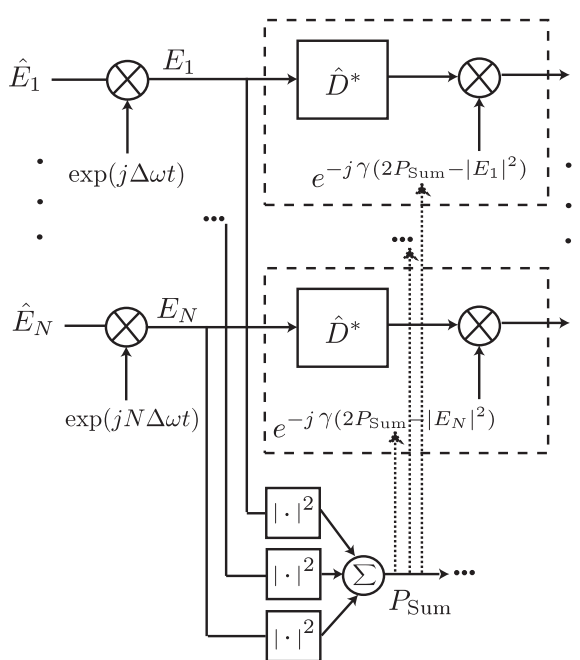

(b) Coupled Field BP

Fig. 1 Diagram of (a) total field back-propagation and (b) coupled field back-propagation. $N$ represents the total number of channels, and $\Delta \omega=2 \pi \Delta f$ represents the channel spacing.

\subsubsection{Coupled field Back-propagation}

The coupled field back-propagation method consists in considering the electric field backward evolution of each band separately. Furthermore, for each backpropagation step all bands are back-propagated in simultaneous, while accounting in the nonlinear phase de-rotation of each band not only the SPM contribution from each band but especially the XPM terms between bands. The mathematical derivation of the cross phase modulation terms is achieved firstly by substituting the expression of the full reconstructed electric field into the NLSE expression given by (1). Secondly, it requires changing the sign of the $\hat{D}$ and $\hat{N}$ operators to reflect the propagation in the backward direction and expanding the $|E|^{2}$ term, while neglecting FWM terms. As demonstrated by Mateo et al. (2008) the resulting expression can be written as:

$$
-\frac{\partial E_{m}}{\partial z}-\hat{D}=j \gamma\left(2 \sum_{i=1}^{N}\left|E_{i}\right|^{2}-\left|E_{m}\right|^{2}\right) E_{m}
$$


where $E_{m}$ represents the electric field of $m$ channel. The right end side of equation (2) represents the nonlinear phase compensation term, which is proportional to twice the instantaneous power summation of the electric fields from all channels, $P_{\text {Sum }}$, as shown in Fig. 1(b). Therefore, due to this simplification, CF BP should be an interesting approach to mitigate SPM and XPM effects, but not FWM.

\section{Computational complexity comparison - coupled field vs total field}

In the context of this paper, it is of relevance to understand the differences in terms of computational complexity between the CF and the TF methods. Mateo et al. (2008) has derived an expression that relates the step size requirements for accurately describing the XPM (for CF) and FWM effects (for TF). He demonstrated that the step size requirements for XPM scale inversely with $\Delta f R_{s}$ while those for FWM scale inversely with $\Delta f^{2}$. Therefore, their ratio scales with $\Delta f / R_{s}$. In fact, this ratio can be related with the number of computations of both approaches, considering that it is inversely proportional to the step size:

$$
\frac{C_{\text {total }}}{C_{\text {coupled }}}=\frac{h_{\text {coupled }}}{N h_{\text {total }}}=\frac{\pi k}{2} \frac{(N-1) \Delta f}{N R_{s}}
$$

where $C$ represents the total number of computations of the algorithm, $R_{s}$ represents the symbol-rate, and $k$ is a constant that depends on the type of algorithm selected. One should note that while $h_{\text {coupled }}$ is inversely proportional to the number of computations per channel, $h_{\text {total }}$ is inversely proportional to the number of computations for all channels, which justifies the introduction of a factor $N$ in equation (3), the total number of channels.

As a means of comparing the performance/complexity of the BP algorithm for both $\mathrm{CF}$ and $\mathrm{TF}$ algorithms, a simulation was performed using the previously shown setup. The average $\mathrm{Q}$ factor among all bands was obtained as a function of the number of BP sections, for different band spacings in the transmitted signal, considering 5 transmitted bands. Although we take the average Q factor among all bands, the FWM impact leads to a different performance being achieved in each band, which is generally worse for the middle bands, as will be explained in section 4. It should be noted that the number of BP sections represents the fixed length of the fiber span $(80 \mathrm{Km})$ divided by the variable step size, such that for 1 section the step size is equal to the length of the fiber span, while for 10 sections the step size is equal to $8 \mathrm{Km}$ of fiber length. The results are shown in Fig. 2.

The number of sections varied from 1 up to 10 for the CF and from 5 to 50 for the $\mathrm{TF}$ algorithm, since that equal complexity is achieved for both algorithms when $h_{\text {coupled }}=N h_{\text {total }}$ as seen in equation (3). We firstly discuss the results of the total field BP, represented in solid lines, while considering a fixed number of sections. It can be found that the performance decreases with increasing channel spacing, since the TF step size requirements $\left(h_{\text {total }}\right)$ are inversely proportional to $\Delta f^{2}$. Therefore, the higher the channel spacing, the higher the required number of sections in order to maintain the same performance, although above 25 sections the difference is negligible. Differently, the CF results show a general improvement tendency with increasing band spacing (for a fixed number of sections). This can be attributed to the higher FWM phenomenon impact for very shortly spaced bands, namely, the impact due to the generation of beating products falling in the neighboring 


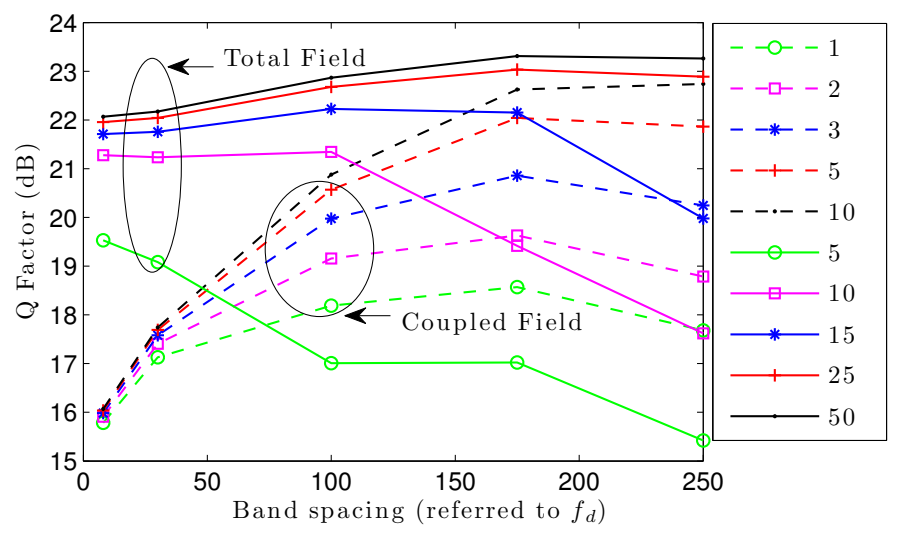

Fig. 2 Q factor versus band spacing, comparing the performance/complexity of the BP algorithm for both $\mathrm{CF}$ and $\mathrm{TF}$ algorithms, considering 5 transmitted bands. Results with equal complexity are depicted with equal markers.

bands. The FWM impact for closely spaced bands can be analyzed in Fig. 3, where the spectra of three distinct signals are shown after forward propagation. It can be concluded that the higher the band-spacing, the lower the amplitude of the distortion products, which can be observed by noting the power spectral density decrease above the noise level, around the spectrum edges.

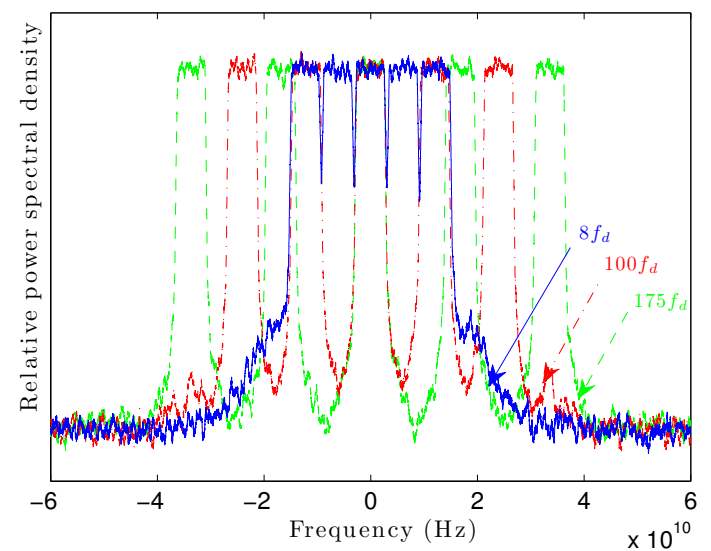

Fig. 3 Power spectral density comparison of three distinct transmitted signals, for a bandspacing of $\left\{8 f_{d}, 100 f_{d}, 175 f_{d}\right\}$, represented in solid, dash-dot and dashed lines, respectively.

Additionally, the reduction of the FWM beating products as the band spacing increases, allows the CF algorithm to show its effectiveness in compensating SPM and XPM effects. Furthermore, the CF step size requirements $\left(h_{\text {coupled }}\right)$ are inversely proportional to $\Delta f R_{s}$, which justifies the need for a higher number of sections as the band spacing increases. This explains the noticeable performance improvement with increasing number of sections as the band spacing increases. 
When comparing both algorithms, one can graphically determine the band-spacing for which both algorithms have equal performance, at the same complexity; this can be found to be a band-spacing of $\{74,168,237\} f_{d}$ for a $\mathrm{Q}$ factor of $\{18$, $19.5,20.5\} \mathrm{dB}$ and for $\{1,2,3\}$ sections in the $\mathrm{CF}$ algorithm, respectively. This means that as we increase the accepted algorithm computational complexity, the band-spacing above which the CF out-performs the TF algorithm also increases. Another parameter of relevance is the required latency of the back-propagation algorithm, since a real-time implementation is likely to be desired. The CF approach is inherently parallelizable, which reduces the system latency compared to the TF method. Mateo et al. (2008) demonstrated that the processing latency ratio between $\mathrm{CF}$ and $\mathrm{TF}$ is inversely proportional to the step size ratio. This means that considering a band-spacing for which both $\mathrm{CF}$ and $\mathrm{TF}$ have the same performance with similar complexity, the processing latency of the TF approach is $N$ times higher. Therefore, assuming that a low band-spacing is desired, although the CF algorithm provides poorer performance than the TF algorithm, it might still be a better alternative based on the allowed reduced processing latency requirements.

\subsection{Analysis for a variable number of bands}

Although in the previous section the $\mathrm{CF}$ and $\mathrm{TF}$ algorithms were compared for similar computational complexity levels, the number of OFDM bands was fixed. However, a higher number of bands could lead to decreased requirements of the receiver analog to digital conversion circuits, while allowing for an increased parallelization of the CF compensation algorithm, which could be desirable. In this sense, it is interesting to understand how the performance of both algorithms is affected due to a higher number of bands. With this objective, we analyze the difference in performance between the CF and TF implementations while using the same computational complexity in both algorithms. We conducted a simulation where the number of bands is varied from 5 up to 25 , while the total symbol rate is kept constant at 25 GSymbols/s, while considering a band spacing of $8 f_{d}$ as shown in Fig. 4 (a) and $100 f_{d}$ as shown in Fig. 4 (b). The solid lines represent a result of similar complexity between both algorithms, since the CF compensation is performed with 1 section per span while the TF is carried with a number of sections equal to the number of bands $(N)$. The results in dash lines show an indication of the performance bound of both algorithms, considering 10 sections for the CF method and 50 sections, for the TF approach. For the CF method, the performance degradation with increasing number of bands is the result of the numerical error increase with the number of bands. An increased error in the estimate of the electric field for each BP step, results from the higher uncertainty achieved in the calculation (summation) of the total instantaneous power, when more bands are considered. The TF method provides improving performance with an increasing number of bands due to the increase of the number of sections used in the algorithm, equal to $N$. It can be concluded that for a small band spacing $\left(8 f_{d}\right)$ the TF approach is better performing for any number of bands. For a higher band spacing ( $100 f_{d}$ in this case) the $\mathrm{CF}$ approach is outperforming only for 5 bands, while the over-performance is not higher than $4 \mathrm{~dB}$ up to 17 bands.

Therefore, from the previous analysis it can be concluded that the $\mathrm{CF}$ algorithm is an interesting option in terms of performance/computational complexity, if the 


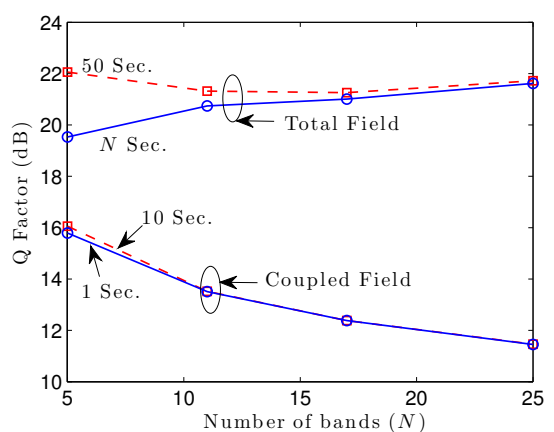

(a) Band spacing $=8 f_{d}$

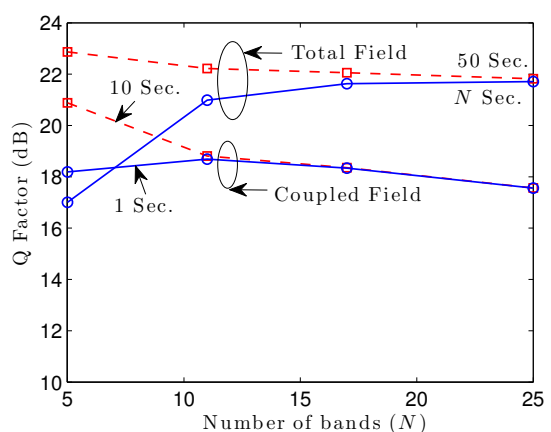

(b) Band spacing $=100 f_{d}$

Fig. 4 Average Q factor versus number of bands for a constant symbol-rate of 25 GSymbols/s, having a band spacing of (a) $8 f_{d}$ and (b) $100 f_{d}$. Solid lines represent a similar computational complexity between both algorithms. Dashed lines represent an approximate performance limit of both algorithms.

system is allowed to have a relatively large band spacing and a reduced number of bands.

\section{Coupled field back-propagation including FWM}

The previous analysis in the expansion of the CF BP equation (2) has not considered the FWM terms. However, if the FWM terms are neglected, there are beating products that are not taken into account in the backward propagation, which leads to a performance degradation for closely spaced bands as seen previously in Fig. 2.

Additionally, the field fluctuations induced from FWM distortion should vary faster than those caused by XPM, which would require a smaller step size to take them into account. Mateo et al. (2008) demonstrated the mathematical derivation of the FWM terms in the nonlinear propagation equation by rewriting the total optical field as:

$$
E=\sum_{m=1}^{N} E_{m} \exp \left(j k_{m} z\right)
$$

where $k_{m}$ is the linear propagation constant of the $m$-th channel. Again, by substituting in the electric field $E$ in equation (1) with the expression for the electric field given by (4), and proceeding in a similar manner as in the derivation of equation (2), Mateo et al. (2008) showed that one can write the nonlinear term, including the FWM part, as follows:

$$
j \gamma\left(2 \sum_{i=1}^{N}\left|E_{i}\right|^{2}-\left|E_{m}\right|^{2}\right) E_{m}+j \gamma\left[\sum_{[r s l m] \in I} E_{r} E_{s} E_{l}^{*} \exp \left(j \delta k_{r s l m} z\right)\right]
$$

with $l=r+s-m$, in order to neglect fast time oscillating terms, and respecting $\{r, s, m\} \in\{\ldots,-2,-1,0,1,2, \ldots\}$ the index of each channel, so that the newly generated waves stay within the WDM band and finally $r \neq s \neq m$ so that SPM and 
XPM terms are not considered twice. The phase mismatch parameter $\delta k_{r s l m}$, influences the efficiency of the FWM, whose maximum intensity is contributed from the edge channels over the central channel. By taking advantage of FWM terms, Mateo and Li (2009) proposed the partial compensation of FWM through the CF method. This consists in using additional terms that reflect the FWM interaction leading to nonlinear mixing between neighboring channels, which are highly phase matched, therefore having the potential to improve the results without compromising the computational complexity. In this way, the FWM terms including the interaction between 2 and 4 neighbors can be found to be, respectively:

$$
\begin{gathered}
F_{2 m}=2 E_{m+1} E_{m-1} E_{m}^{*} \\
F_{4 m}=E_{m+1}^{2} E_{m+2}^{*}+E_{m-1}^{2} E_{m-2}^{*}+2 E_{m-1} E_{m+2} E_{m+1}^{*} \\
+2 E_{m+1} E_{m-2} E_{m-1}^{*}+2 E_{m+2} E_{m-2} E_{m}^{*}
\end{gathered}
$$

Here, the phase mismatch exponential has been omitted for simplicity. Therefore, the nonlinear term in the BP equation can be written in the following simplified manner:

$$
j \gamma\left(2 P_{\text {sum }}-\left|E_{m}\right|^{2}\right) E_{m}+j \gamma\left(F_{2 m}+F_{4 m}\right)
$$

Additionally, Mateo and Li (2009) suggests a perturbative implementation to solve equation (8), such that the nonlinear operator for step $i$ is approximated as:

$$
E_{m}^{i+1}=E_{m}^{i} \exp \left[j \gamma h\left(2 P_{\text {sum }}-\left|E_{m}\right|^{2}\right)\right]+\gamma h\left(F_{2 m}^{i}+F_{4 m}^{i}\right)
$$

where $h$ represents the BP step size. However, this approximation is only valid if the number of neighboring channels considered for FWM compensation is a small fraction (below $20 \%$ ) of the total number of channels interacting through $\mathrm{XPM}$. This is required so that the phase mismatch length becomes larger than the walk-off length, implying that field variations due to FWM are slower than the variations due to XPM. However, this could preclude the usage of this algorithm when the number of transmitted bands is small, therefore we propose a solution in the next section.

\subsection{Partial FWM compensation method for multi-band OFDM signals}

In this work, a novel strategy of implementing a partial FWM compensation scheme is proposed. Instead of performing the perturbative compensation as suggested in Mateo and Li (2009), which is suitable for WDM transmission with a large number of channels, equation (9) is modified in order to reduce the numerical error, due to a small number of bands being considered. We propose to compute the BP step as follows:

$$
E_{m}^{i+1}=E_{m}^{i} \exp \left[-j \zeta \gamma L_{\mathrm{eff}}\left(2 P_{\mathrm{sum}}-\left|E_{m}^{i}\right|^{2}\right)\right]+\xi \gamma L_{\mathrm{eff}}\left(F_{2 m}^{i}+F_{4 m}^{i}\right)
$$

where $L_{\text {eff }}$ represents the fibre effective length (smaller than L due to fibre losses Agrawal (2001)), $\zeta$ represents the nonlinear phase rotation parameter (as proposed in Ip and Kahn (2008)) and $\xi$ represents an empirical parameter proposed here. This parameter is intended to control the amount of FWM compensation, its optimum value, between 0 and 1, being found through numerical optimization. 
The average Q factor performance using the new partial FWM compensation method with the coupled field approach for a signal with 5 bands is shown in Fig. 5 , for two different band spacings. Specifically, Fig. 5(a) is obtained for a band spacing of $30 f_{d}$ and Fig. 5(b) for a band spacing of $50 f_{d}$, considering the interaction of both 2 and 4 neighbor bands.

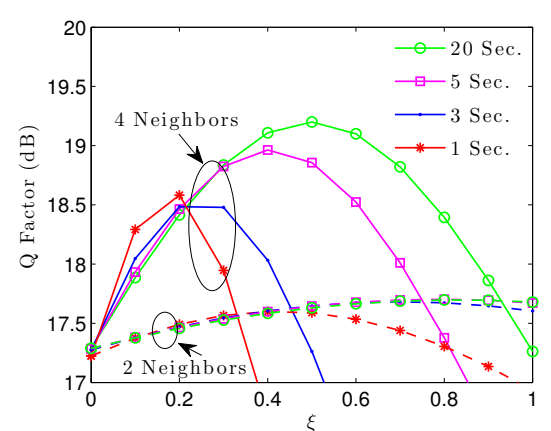

(a) Band spacing $=30 f_{d}$

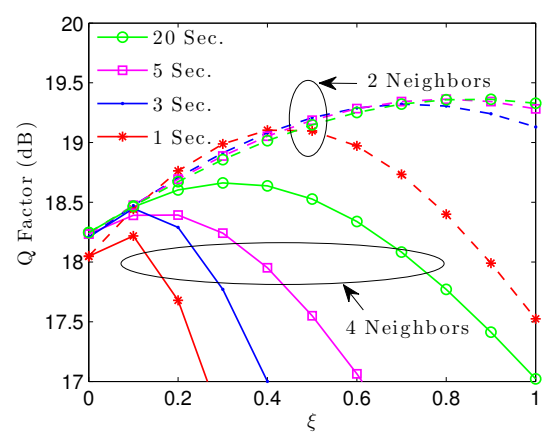

(b) Band spacing $=50 f_{d}$

Fig. 5 Q factor versus $\xi$, for bands spaced at (a) $30 f_{d}$ and (b) $50 f_{d}$, compensating the FWM interaction of 2 neighbors (dashed lines) and 4 neighbors (solid lines).

From the analysis of Fig. 5, it can be seen that the proposed algorithm effectively provides some performance gain, compared to using only the coupled field algorithm, which corresponds to the performance at the $\xi=0$ point. Additionally, the higher the number of sections used in the BP algorithm, the higher the performance gain, although we observed that above 20 sections the improvement is negligible. Additionally, the optimum $\xi$ parameter depends on the type of FWM compensation used ( 2 or 4 neighbors) and increases with the number of sections used, becoming constant above 20 sections. In practice, the $\xi$ parameter can be adjusted after each BP step, therefore not compromising the latency of a desired parallel real-time implementation.

The algorithm performance improvement relative to the simple CF method was assessed as a function of the the band spacing, considering a variation from $8 f_{d}$ up to $200 f_{d}$. The results are shown in Fig. 6 . The $\xi$ parameter used in the simulations corresponds to the optimum value found for each case. The results show that the advantage of using the partial FWM compensation is more effective for a band spacing around $30 f_{d}$, gradually vanishing as the band spacing increases, regardless of the number of sections used in the $\mathrm{BP}$ algorithm. The reason behind this behavior is related with the effect of FWM impairment itself, whose strength decreases as the channels are further separated from each other. However, for the range of band spacings where FWM has an impact, Mateo et al. (2008) has pointed out that the intensity of FWM induced field variations is essentially due to the interaction of signals more distant in frequency. This is consistent with the fact that the amplitude of the provided performance improvement considering the interaction between 2 neighbors is more significant for a band spacing around $75 f_{d}$, whereas for the interaction between 4 neighbors it is more significant for a band spacing around $30 f_{d}$. 


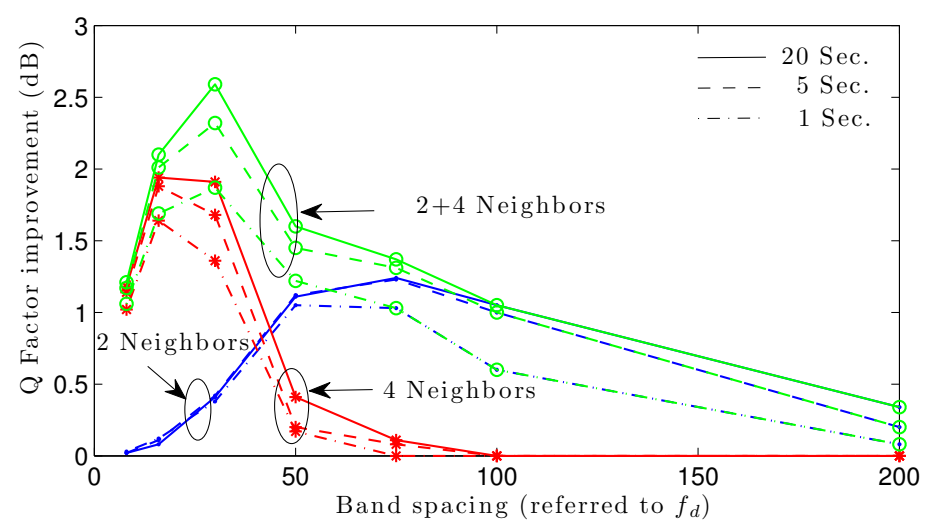

Fig. 6 Q factor versus band spacing, for the interaction of 2 neighbors (blue lines), 4 neighbors (red lines) and the combination of both (green lines), for $\{1,5,20\}$ sections in the asymmetric BP algorithm.

For the specific case of band spacing equal to $30 f_{d}$, Fig. $7($ a) shows the performance improvement obtained for each band, comparing the case of using the coupled field algorithm only with that of using the coupled field with FWM compensation. The cases of including 2 neighbor bands, 4 neighbor bands and the combination of both were considered, the $\mathrm{Q}$ factor improvement being $0.4 \mathrm{~dB}$, $1.38 \mathrm{~dB}$ and $1.72 \mathrm{~dB}$, respectively. Note that the 4 neighbor FWM result only shows improvement for the central band, which is the only band having 4 interacting neighboring bands.

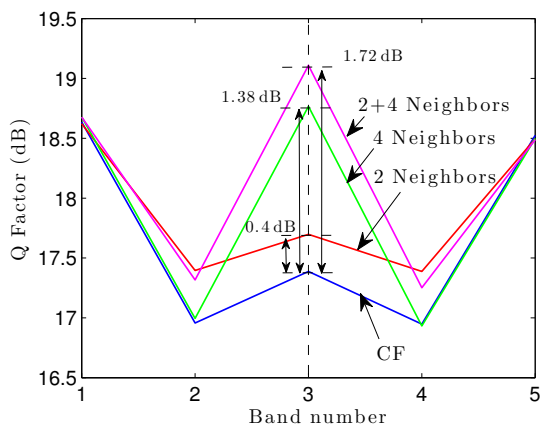

(a) Band spacing $=30 f_{d}$

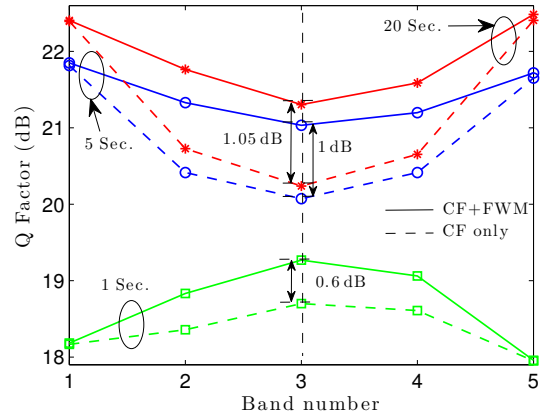

(b) Band spacing $=100 f_{d}$

Fig. 7 Q factor versus band number comparing the performance of using only the coupled field algorithm with that of using the coupled field algorithm in combination with the partial FWM compensation, showing (a) impact of using $\{2,4,2+4\}$ neighbors for the compensation and (b) different number of sections.

The impact of varying the number of sections is shown in Fig. 7(b) for a band spacing of $100 f_{d}$, comparing the relative performance of using $\{1,5,20\}$ sections in the BP algorithm. The dashed lines represent the result of using the coupled field method alone, while the solid lines represent the result of using the coupled 
field method combined with the FWM compensation of 2 neighbor channels. As shown, the performance increase affects not only the central band but also the two neighboring bands where the algorithm is also applied. The improvement increases with the number of sections, improving from $0.6 \mathrm{~dB}$ up to $1.05 \mathrm{~dB}$, for an increase from 1 to 20 sections, which confirms that the FWM compensation effectively becomes more precise due to the numerical error reduction when the number of sections is increased. It should be noted that the performance of the center bands tends to be worse than those bands in the edge, due to the fact that the middle ones suffer from stronger FWM, since they have a higher number of neighbors. Mateo and Li (2009) reported a performance gain up to $1.5 \mathrm{~dB}$ for the center channels of a $24 \mathrm{WDM}$ channel setup using 16-QAM. In the present work, performance improvements above $2 \mathrm{~dB}$ were observed, depending on the band spacing considered, for the case of five closely spaced OFDM bands.

\section{Conclusions}

We analyzed the back-propagation algorithm for OFDM multi-band transmission focusing on the comparison between coupled field and total field back-propagation. These algorithms have been compared based on computational complexity where it was concluded that the coupled field algorithm is an interesting option, especially for large band spacings and a reduced number of bands, since for small band-spacings the CF method is not able to compensate the degradation induced by FWM. Additionally, a FWM compensating term was considered within the coupled field method to provide simplified means of multichannel nonlinearity compensation for multi-band OFDM signals. A compensation method was proposed in this context, which allows for a considerable performance improvement when using a relatively small band spacing. This is the case where FWM induced field variations are more intense, making the $\mathrm{CF}$ method an interesting choice for the implementation of receiver-based real-time digital signal processing of future coherently detected long-haul optical links.

\section{References}

Agrawal G (2001) Nonlinear Fiber Optics, 3rd edn. Academic Press

Chowdhury A, Essiambre R (2004) Optical phase conjugation and pseudolinear transmission. Optics letters 29(10):1105-1107

Hasegawa A, Tappert F (1973) Transmission of Stationary Nonlinear Optical Physics in Dispersive Dielectric Fibers I: Anomalous Dispersion. Appl Phys Lett 23(3):142-144

Hayee Q (2008) Symmetrized split-step Fourier scheme to control global simulation accuracy in fiber-optic communication systems. J Lightwave Technol 26:302-316

Ip E, Kahn J (2009) Nonlinear impairment compensation using backpropagation. In-Tech, Vienna Austria

Ip E, Kahn JM (2008) Compensation of Dispersion and Nonlinear Impairments Using Digital Backpropagation. Journal of Lightwave Technology 26:3416-3425, DOI 10.1109/JLT.2008.927791 
Li X, Chen X, Goldfarb G, Mateo E, Kim I, Yaman F, Li G (2008) Electronic post-compensation of WDM transmission impairments using coherent detection and digital signal processing. Opt Express 16:880-888

Lowery A (2007) Fiber nonlinearity pre-and post-compensation for long-haul optical links using OFDM. Opt Express 15:12,965-12,970

Mateo E, Li G (2009) Compensation of interchannel nonlinearities using enhanced coupled equations for digital backward propagation. Applied optics 48(25):F6F10

Mateo E, Zhu L, Li G (2008) Impact of XPM and FWM on the digital implementation of impairment compensation for WDM transmission using backward propagation. Optics Express 16(20):16,124-16,137

Roberts K, Li C, Strawczynski L, O'Sullivan M, Hardcastle I (2006) Electronic precompensation of optical nonlinearity. IEEE Photonics Technology Letters 18(2):403-405

Shieh W, Yang Q, Ma Y (2008) 107 Gb/s coherent optical OFDM transmission over 1000-km SSMF fiber using orthogonal band multiplexing. Opt Express 16(9):6378-6386 